\title{
A REDUNDANT GNSS-INS LOW-COST UAV NAVIGATION SOLUTION FOR PROFESSIONAL APPLICATIONS
}

\author{
J. Navarro ${ }^{\mathrm{a}}$, M.E. Parés ${ }^{\mathrm{a}}$, I. Colomina ${ }^{\mathrm{b}}$, G. Bianchic, S. Pluchino ${ }^{\mathrm{c}}$, R. Baddour ${ }^{\mathrm{d}}$, A. Consoli ${ }^{\mathrm{e}}$ J. Ayadie, A. Gameiro ${ }^{\mathrm{f}}$, O. Sekkas $^{\mathrm{g}}$, \\ V. Tsetsos ${ }^{\mathrm{g}}$, T. Gatsos ${ }^{\mathrm{g}}$, R. Navoni ${ }^{\mathrm{h}}$ \\ ${ }^{a}$ CTTC. Av. Carl Friedrich Gauss 7 - Building B4, E-08860 Castelldefels, Spain - (jose.navarro, eulalia.pares)@cttc.es \\ ${ }^{\text {b} G e o N u m e r i c s, ~ S . L ., ~ A v . ~ C a r l ~ F r i e d r i c h ~ G a u s s ~} 11$ - Building B6, E-08860 Castelldefels, Spain - ismael.colomina@geonumerics.com \\ 'Istituto Nazionale di Astrofisica - Istituto di Radioastronomia. Via Fiorentina 3513, 40059 Medicina, Italy - (g.bianchi, \\ s.pluchino)@ira.inaf.it \\ dSaphyrion Sagl. Strada Regina 16, CH-6934 Bioggio, Switzerland - rami.baddour@saphyrion.ch \\ eEclexys Sagl. Via dell’inglese 6, CH-6826 Riva San Vitale, Switzerland - (angelo.consoli, jaouhar.ayadi)@eclexys.com \\ fBrightcom lda. Rua Afonso Lima 8, F. Foz, Portugal - amg@ua.pt \\ gMobics S.A. Kifisias 27, 11523, Athens, Greece - (sekkas, btsetsos, tgatsos)@mobics.gr \\ hLaser Navigation SRL, Via Matteotti 34, 24054 Calcio (BG), Italy - lasernav@gmail.com
}

KEY WORDS: UAV, reliable navigation, redundant IMUs, GNSS beamforming, close coupling

\begin{abstract}
:
This paper presents the current results for the FP7 GINSEC project. Its goal is to build a pre-commercial prototype of a low-cost, accurate and reliable system for the professional UAV market. Low-cost, in this context, stands for the use of sensors in the most affordable segment of the market, especially MEMS IMUs and GNSS receivers. Reliability applies to the ability of the autopilot to cope with situations where unfavourable GNSS reception conditions or strong electromagnetic fields make the computation of the position and / or attitude of the UAV difficult. Professional and accurate mean that, at least using post-processing techniques as PPP, it will be possible to reach cm-level precisions that open the door to a range of applications demanding high levels of quality in positioning, as precision agriculture or mapping. To achieve such goal, a rigorous sensor error modelling approach, the use of redundant IMUs and a dual-GNSS receiver setup, together with close-coupling techniques and an extended Kalman filter with self-analysis capabilities have been used. Although the project is not yet complete, the results obtained up to now prove the feasibility of the aforementioned goal, especially in those aspects related to position determination. Research work is still undergoing to estimate the heading using a dual-GNNS receiver setup; preliminary results prove the validity of this approach for relatively long baselines, although positive results are expected when these are shorter than $1 \mathrm{~m}$ - which is a necessary requisite for small-sized UAVs.
\end{abstract}

\section{INTRODUCTION}

The goal of the European Community's Seventh Framework Program (FP7) Enhanced GNSS-BF-INS Solution for Unmanned Vehicle Control (GINSEC) project is to build a precommercial prototype of a low-cost, accurate and reliable navigation system for the professional UAV market. Current low-cost navigation systems for UAVs are still affected by many practical problems as the slow time to first fix (up to a few minutes), poor or no availability of GNSS signal, slow dynamics and poor accuracy as well as the lack of a reliable heading indication. These problems may be critical to professional users. GINSEC aims at developing a navigation system that should solve these problems with various sensor configuration and fusion approaches: redundant low-cost Inertial Measurement Units (IMU) to improve dynamics and availability of navigation, using a closely coupled approach and redundant GNSS antennas, to obtain heading estimation.

The GINSEC consortium consists of Small \& Medium Enterprise (SMEs) and Research \& Technological Development (RTD) performers that are active in the GNSS/INS market. RTD partners are involved in the selection and characterization of the low cost sensors (deriving their error models) and in the study and development of the data fusion algorithms for navigation; the SMEs will develop, manufacture and test the prototype navigation system. The challenge is to implement and integrate all above technologies in the frame of limited size, weight and cost imposed by the small-size UAV market requirements. Through their collaboration, the partners aim to develop a navigation solution directly exploitable on various kinds of small-size UAVs.

\section{WHERE FROM? WHERE TO? GOAL, CHALLENGES AND PROPOSED ANSWERS}

\subsection{Where from?}

Laser Navigation, one of the SMEs integrating the consortium, already commercializes a navigation system for low-cost UAVs. Their product, the VRBrain autopilot system, is built around the VRBrain multipurpose board and the open source APM:Copter software (the specific version of the APM autopilot for multicopter platforms.) Such solution relies essentially on a GNSS receiver, a single IMU and a magnetometer. The IMU is used to estimate pitch and roll as well as position between GNSS updates. Heading is obtained from the magnetometer. From the sensors standpoint, no redundancy is provided. This configuration is heavily affected by multipath issues and electromagnetic interferences (i.e. when approaching power lines). The heading determination is critically sensible to these problems and this compromises both the quality and reliability of the navigation solution. The lack of reliability has a direct impact on the ability of the UAV to navigate autonomously. On the contrary, low-quality navigation solutions clash over the requirements of the applications to be exploited in such UAVs, which may need much higher precisions than those provided by the current configuration.

\subsection{Where to? The Goal}

Shortly stated, the goal of GINSEC is to overcome these limitations, providing a professional-grade solution relying on redundant low-cost GNSS and MEMS IMU sensors, to improve both the autopilot and the quality of the products that may 
eventually be generated by the different kinds of payloads carried by the UAV.

In the context of the GINSEC project, this means that:

- the autopilot should be able to better cope with situations where the conditions for GNSS reception are not optimal, as multipath, urban canyons, or bridges;

- the same applies to scenarios with high electromagnetic interferences where magnetometers are heavily affected, thus delivering incorrect heading readings.

- The quality of the navigation solution must be higher than the one provided by the current solution in terms of position and attitude precision.

The precision of the navigation solution needs not to reach the cm-level - usually demanded by professional applications - in real time; that is, such requirement does not apply to the autopilot itself. However, this precision must be achievable by - at least post-processing the navigation data logged by the system (see section 2.4) so the quality of the products generated by the payload may reach professional levels.

\subsection{Challenges and Proposed Answers}

The aforementioned objective implies several immediate challenges, the first of these being the suitability of low-cost sensors to provide with data with the needed quality. Other evident challenge is how to guarantee that the combination of such data will lead to an acceptable navigation solution that, additionally, must be reliable enough as to be trusted in all situations.

The foreseen answers to these problems are, among others, redundancy, heterogeneity and rigorous (sensor) modelling - the usual pillars of Geodesy - as well as the use of a solution estimation method that pays special attention to reliability (an extended Kalman filter with quality self-analysis tools.)

In the context of this paper, redundancy stands for the availability of multiple sources providing the same type of data, as for instance two GNSS receivers (Mendes, 2012). Note, however, that for the GINSEC project redundancy does not play the role of a fallback mechanism - use a spare receiver when the other fails - but a way to increase the amount of data collected to compute the navigation solution and thus, to reduce noise.

Heterogeneity stands for the use of different types of data sources contributing to the estimation of the solution (Groves, 2013). The use of a close-coupling approach to combine GNSS and IMU data plus the geometric restrictions set by the distribution of the several sensors over the UAV are also mechanisms to achieve the stated goals.

Rigorous modelling is the determination of the accurate models describing both the properties and errors of the data collected by those (El-Sheimy, 2008).

\subsection{Benefits: Technical and Economical}

From the technical standpoint, the impact (and related benefit) of such an improved solution intends to be twofold.

${ }^{1}$ The price given usually include not only the UAV itself, but also the control station as well as some kind of software to process the data collected and generate some kind of product. It is not therefore clear how much of this cost corresponds to
First, the autopilot responsible for controlling the flight of the UAV will be much better suited to face the challenges that autonomous navigation poses. For instance, the availability of redundant IMU data plus a close-coupling approach to integrate GNSS data increases the quality - which may be assessed on real time - of the estimation for both position and attitude. The tandem magnetometer - dual-GNSS receiver setup provides also with a more reliable heading estimation.

On the other side, post-processing the navigation data collected by the different sensors integrated in the platform using well know techniques as Point Precise Positioning (PPP) yields results that show centimetre-level precision. Since PPP does not depend on the availability of Continuously Operating Reference Stations (CORS) the operational range of the UAV is not compromised. When working on campaigns where the operational range is limited and CORS are available, the Point Precise Kinematic (PPK) technique may be used yielding also results in the same precision range.

Both post-processing approaches have a direct impact on the quality of the product derived from the data captured by the payload - whatever it is - carried by the UAV. For instance, a photogrammetric flight may benefit from such post-processing in the sense that the logistics needed to guarantee quality results would be far simplified - requiring, among other things, less ground control points, which are relatively costly to set up.

Post-processing is clearly beneficial for the quality of the target product, but has no impact on the navigation solution used by the autopilot. However, when CORS are available and it is affordable to install a radio link is on the UAV, it is possible to use the Real Time Kinematic technique (RTK) to obtain the aforementioned precisions on real time and still better the behaviour of the autopilot.

From the economical point of view, decreasing the cost of a UAV able to provide a professional-grade navigation solution not only for the autopilot but also for the processing of the data captured by the payload may have noticeable consequences.

Small companies willing to enter the market have to face the sometimes unsurmountable barrier of the price of such platforms. UASs sold nowadays by well know companies are priced around 25.000 to $30.000 €^{1}$.

Reducing noticeably the price of the UAV itself may help these companies to decide on entering any market where these platforms are suitable for generating value-added products.

\section{THE PROTOTYPE}

As stated above, the main challenges the GINSEC project had to deal with where:

- Finding a suitable set of sensors (specially IMUs) able to provide observation data good enough to compute a professional-grade navigation solution and,

- Obtain a reliable heading estimation using a dual-antenna GNSS receiver setup, to avoid the need to rely on a magnetometer.

the UAV itself, so comparing the price of these solutions with the cost of the prototype described here may prove at least imprecise. 
The following sections discuss on these two points above. Additionally, the strategy adopted to validate results as well as the actual implementation of the different intervening algorithms on the target platform are described as well.

\subsection{Finding the Right Sensors}

Among all the sensors used by the VRBrain platform used as the starting point for the GINSEC project, only the IMUs were considered to be deeply investigated.

Magnetometers were left out of the study, since the new approach to determine heading would use these mainly to obtain an initial approximation of this value before starting the flight. Once flying, magnetometer readings are used as a redundant way to test the quality of the heading estimation obtained by a dual-GNSS setup, and may be rejected if its values clearly disagree with the values obtained using the last method.

Concerning GNSS receivers, little room for manoeuvring was available; from the GINSEC standpoint, these may be roughly classified in two groups: those with a high-level performance and noticeable weight and cost and the set of small, lightweight, much cheaper ones. The goals of the project directly imposed the use of receivers in this last group; since these show a very similar set of features and performance, the decision was to incorporate the uBlox 8, a brand already used in the VRBrain platform.

It is important to note, however, that the GNSS receiver had to be able to provide raw observation data besides the position usually computed by these, because, otherwise, it would not have been possible to apply the close-coupling technique to improve the navigation solution.

IMUs were a very different matter. In the original VRBrain implementation, only one IMU was used, primarily to provide with pitch and roll estimations as well as to help to determination of the position between GNSS updates. The new approach uses data output by the IMUs, together with GNSS updates, to compute a reliable navigation solution by means of a closely coupled technique. Therefore, the quality of the selected IMUs was a matter to take into account.

A market survey was then conducted to investigate the characteristics of the MEMS IMUs available at that moment. The requirements derived from the target applications of GINSEC were used as the criterion to select the ones best fitting the needs of the project.

Two candidates were selected, the Invensense MPU6500 and the MAXIM MAX21100. A series of data acquisition campaigns took place to analyse the behaviour of the errors affecting the data thus collected. Redundancy was achieved setting up dual-IMU configurations for each model and test.

Additionally, an extra IMU of - presumably - better quality and higher price, the EPSON S4E5A0A0A1 was also included in the tests as a reference unit. The tests included both static and dynamic observations, to analyse the behaviour of the IMUs in different situations.

The Allan variance analysis was used to model the behaviour of the errors. Such analysis served to obtain the error models for both IMUs, to compare their performance with the reference, higher quality one and finally to select the best one - according to the obtained results. The selected IMU was the MAXIM MAX21100 since it showed better noise characteristics than the
Invensense MPU6500. Comparing the MAXIM MAX21100 to the reference EPSON IMU revealed very promising - and partly quite unexpected - results.

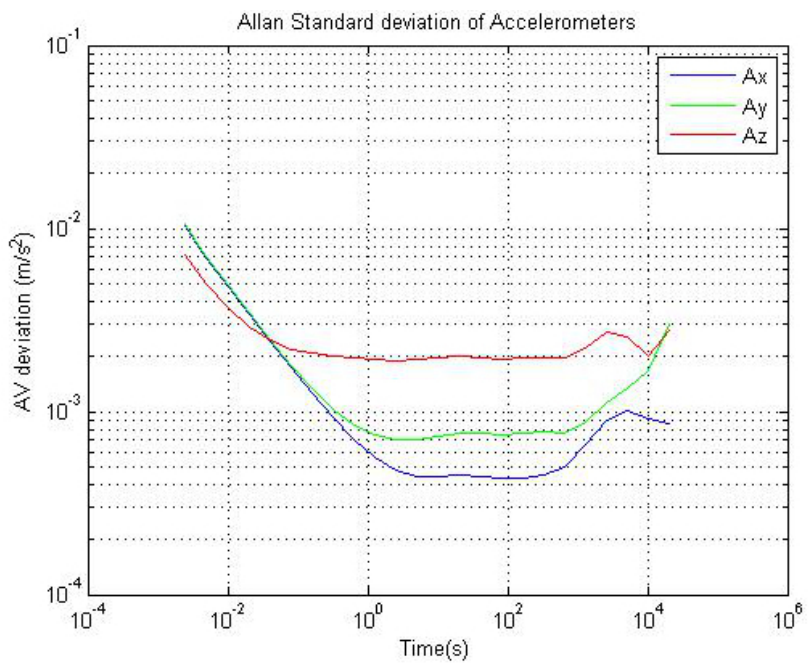

Figure 1: Allan variance for the EPSON accelerometers

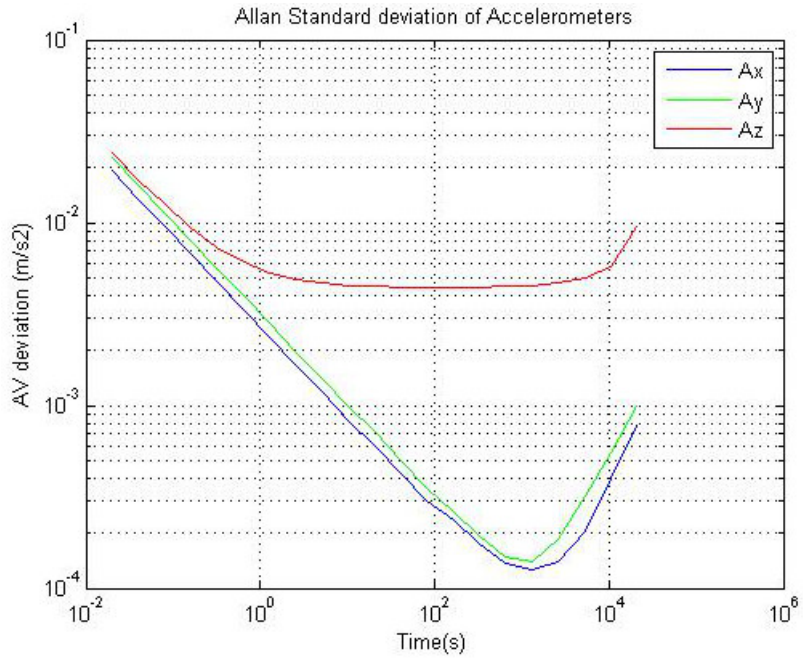

Figure 2: Allan variance for the MAXIM accelerometers

\begin{tabular}{|l|l|}
\hline \multicolumn{2}{|c|}{ EPSON S4E5A0A0A1 specifications } \\
\hline \multirow{2}{*}{ Gyro in-run bias stability } & $5.4 \mathrm{deg} / \mathrm{h}$ \\
& 1 sigma \\
Gyro noise density & $0.002 \mathrm{deg} / \mathrm{s} / \mathrm{sqrt}(\mathrm{Hz})$ \\
& $\mathrm{RMS}, \mathrm{f}=125 \mathrm{~Hz}$ \\
Accel. in-run bias stability & $0.1 \mathrm{mG}$ \\
& 1 sigma \\
Accel. noise density & $0.06 \mathrm{mG} / \mathrm{sqrt}(\mathrm{Hz}) ;$ \\
& RMS, f = 125 Hz \\
\hline
\end{tabular}

Table 3: Specifications for the EPSON IMU

Figure 1 and Figure 2 show the Allan variance plots for the accelerometers of the EPSON and MAXIM IMUs respectively. Table 3 and Table 4 include the results derived from these figures. Here it is possible to see that although the price 
difference of both IMUs amounts to about three orders of magnitude, the performance differs only in one order of magnitude. The results of the IMU characterization campaigns seem to confirm the viability of the GINSEC approach. The suitability of these results is discussed later on this document.

\begin{tabular}{|l|l|}
\hline \multicolumn{2}{|c|}{ MAXIM MAX21100 specifications } \\
\hline Gyro in-run bias stability & $7.2 \mathrm{deg} / \mathrm{h}$ \\
& 1 sigma \\
Gyro noise density & $0.002 \mathrm{deg} / \mathrm{s} / \mathrm{sqrt}(\mathrm{Hz})$ \\
& $\mathrm{RMS}, \mathrm{f}=50 \mathrm{~Hz}$ \\
Accel. in-run bias stability & $5 \mathrm{mG}$ \\
& 1 sigma \\
Accel. noise density & $30 \mathrm{mG} / \mathrm{sqrt}(\mathrm{Hz})$ \\
& $\mathrm{RMS}, \mathrm{f}=50 \mathrm{~Hz}$ \\
\hline
\end{tabular}

Table 4: Specifications for the MAXIM IMU

\subsection{Solving the Heading Problem}

This section presents a short summary of the work by D. Zhen (Zhen, 2008) on which the approach to solve the heading problem in the context of the GINSEC project is based. This is done just for the sake of completeness. A noticeable difference, however, is that GINSEC intends to use Zhen's approach in real time to estimate heading, while the original work is targeted at post-processing.

The idea is to control the flight direction through antennas installed on the UAV. These interact with the GNSS satellites and are not affected by magnetism, eliminating the risk induced by incorrect magnetometer readings. Using two antennas (see Figure 5) it is possible to determine the heading (yaw movement).

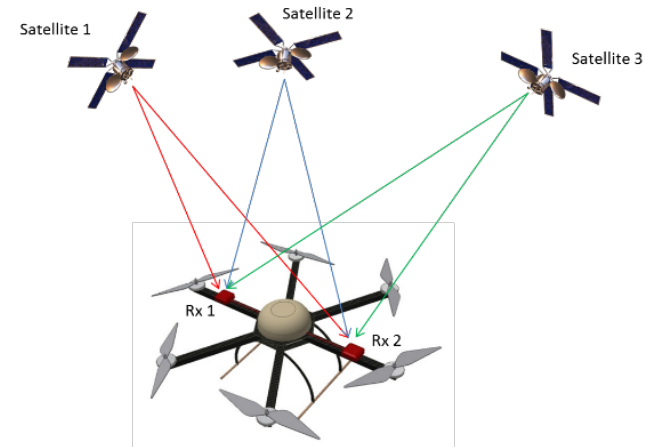

Figure 5: Short baseline antennas installed on the UAV to estimate the orientation

The heading determination uses two receivers with antennas separated by a given short baseline ${ }^{2}$ to estimate the orientation of the UAV. The multi-antenna schematic block is depicted in Figure 6.

In order to compute attitude parameters using a dual-GNSS receiver system, two set of coordinates are needed. The first is referred to a local reference frame; the other one refers to an antenna body frame. The origin of the local reference frame is

2 The lever arm may be calibrated in the laboratory. However, even if such calibration does not exist, the direct attitude computation is still able to yield acceptable attitude results. located at the centre of the first antenna; the coordinates of the centre of the second antenna are computed by GNSS measurements. The antenna body frame coordinates $b(0, L, 0)$ are assumed to have been initially determined by a laboratory calibration process and to remain unchanged. If both the body frame $b(0, L, 0)$ and the local frame GNSS coordinates $u\left(x_{2}, y_{2}, z_{2}\right)$ of the secondary antenna are known, it is possible to solve the system $b=R(\psi, \theta, \varphi) \cdot u$, where $R$ is the heading $(\psi)$, pitch $(\theta)$ and roll $(\varphi)$ rotation matrix.

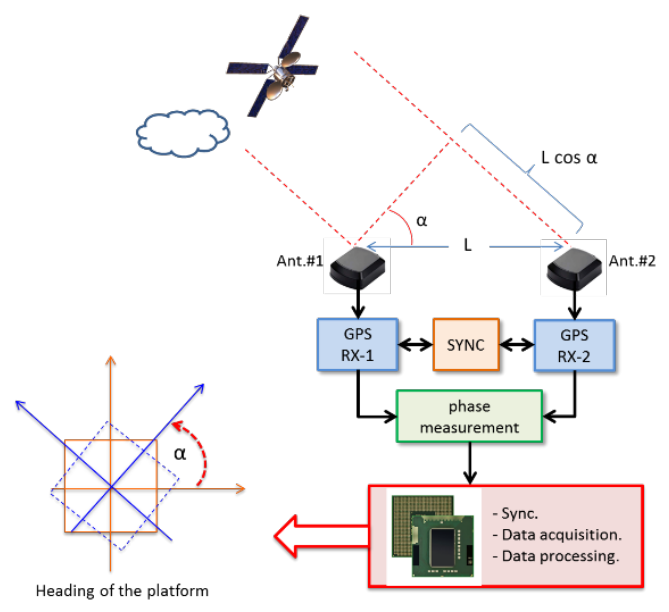

Figure 6: Multi-antenna schematic block

If two or more baseline vectors are available (i.e. in a threeantenna system), the attitude parameters can be estimated by an implicit least-squares model; when only one baseline is at hand, the direct computation model may be used. For a dual-antenna system, the least-square estimation yields results identical to the direct computation method.

Using the orthogonality of the attitude matrix, the formulae for direct computation are:

$$
\begin{gathered}
\psi=\tan ^{-1}\left(\frac{\Delta_{E}}{\Delta_{N}}\right) \\
\theta=\tan ^{-1}\left(\frac{\Delta_{U p}}{\sqrt{\Delta_{E}^{2}+\Delta_{N}^{2}}}\right)
\end{gathered}
$$

Where $\Delta_{E}, \Delta_{N}, \Delta_{U p}$ are respectively the three components of the baseline vector between the two antennas, determined from GNSS in north, east and vertical directions in the local frame.

Carrier phase measurements are essential for reliable heading and pitch determination using GNSS, especially for short baselines. The carrier phase measurements may be collected at equally spaced nominal epochs as the sum of the total number of full carrier cycles plus a fractional cycle between the GNSS satellite and the receiver (see Figure 7). However, the GNSS receiver is unable to distinguish one cycle from another, and the unknown number of these is known as the carrier phase "integer ambiguity", which must be determined along with other unknowns by means of integer ambiguity resolution methods. Due to multipath and the dispersive nature of ionosphere - which causes variations in the speed of carrier propagation - the noise 
of measurements differs among receivers. A common method to reduce this error is to use differential techniques. The combination of measurements relative to the same satellite collected simultaneously by two receivers, eliminates the satellite clock offset and reduces orbital and atmospheric errors. This process is known as Single Differencing (SD) method. This SD technique may be applied to all visible satellites obtaining a set of SD measurements. The combination of these SD measurements is called Double Differencing (DD) (Groves, 2013). DD cancels or reduces to acceptable levels the clock offset and biases, the multipath and the ionospheric effects on the phase measurements.

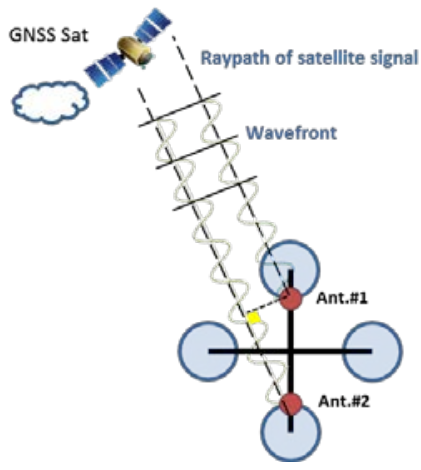

Figure 7: Receiving a waveform from two separated antennas

The selected approach for GINSEC project is Double Differencing. For more information, see (Consoli, 2015).

\subsection{Heading Simulation}

Different measures on the field have been carried out to test the concept described above. Three GNSS receivers were used, working with different baselines ranging from tens of meters down to sub-meter distance. The basic performance was evaluated by means of Double Differencing, using a MATLAB toolbox (Zhen, 2008). In particular, starting from three RINEX files obtained from three GNSS receivers, an accurate computation of attitude parameters (yaw, pitch and roll) as well as their accuracy were obtained.

Simulations have been done using real data acquired from a GNSS setup where three receivers were mounted with baselines of $10 \mathrm{~m}, 1 \mathrm{~m}, 50 \mathrm{~cm}$ and $20 \mathrm{~cm}$. With a baseline of $10 \mathrm{~m}$, the heading accuracy was better than 2 degrees with a standard deviation of about 0.4 degree. Shorter baselines showed some decreased accuracy, that may be related to the receivers' clock noise and to the lack of a coherent clock reference; the consortium is currently investigating the situation and working on its improvement.

\subsection{The Navigation Solution}

Once that all measurement data (GNSS, redundant IMUs, heading) were available and properly characterized, it was the time to select the navigation solution algorithm. The available options were to perform either a loose-, close- or tight-coupling integration of these data.

\footnotetext{
${ }^{3}$ For more information about GEMMA, see the Appendix at the
} end of this document.
Although well known (Groves, 2013), a short description on these three approaches is given below just for the sake of completeness.

Loosely coupled algorithms use GNSS positions as well as IMU and heading data to compute position, velocity and attitude; IMU systematic errors are also estimated and used to correct IMU's measurements -there is a feedback loop. In a sufficiently good GNSS scenario, the solution will not drift.

Closely coupled algorithms use GNSS raw data instead of positions to compute, together with the IMU and heading data, the position, velocity, attitude and IMU systematic errors. As it happens with loosely coupled algorithms, these errors are fed back into the IMU to correct its measurements. This kind of integration allows for GNSS updates even in non-friendly scenarios.

A tightly coupled algorithm is a step forward, since not only the IMU measurements are corrected but also the GNSS receiver is fed back with relevant information (Doppler estimation) that will further improve satellite tracking.

GINSEC uses a closely coupled approach with redundant IMUs (Colomina, 2004), since low-cost commercial GNSS receivers usually do not allow for Doppler corrections. It is important to mention that the GNSS data processing algorithms used in a closely coupled hybridization techniques are exactly the same than those used in "pure" GNSS processing (DGPS, RTK, PPP, etc.).

\subsection{Validating the Navigation Solution}

This navigation solution had to be validated and its performance compared to the requirements set by the target applications of the GINSEC project. To do it, CTTC's GEMMA (Generic Extensible Modular Multi-sensor navigation Analysis system $)^{3}$ was used.

The first step was to create some reference trajectories; to do it, both GEMMA trajectory generators tools as well as a very advanced flight simulator, X-plane 10 , were used. Figure 8 depicts one of the high-dynamics trajectories created with the flight simulator.

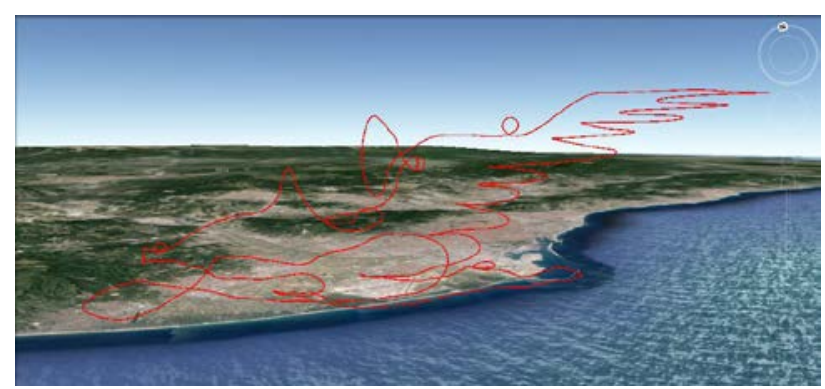

Figure 8: Simulated high-dynamics, aerobatic trajectory

These reference trajectories were used by GEMMA to generate the ideal signals the sensors involved in such flights would have produced; these signals were distorted using the error models obtained in the sensor characterization phase. The result was a dataset including simulated observations from the intervening sensors mimicking the real ones as much as possible. 
These datasets were then processed by GEMMA's trajectory determination tool, NAVEGA, which used the aforementioned sensor error models to compute the navigation solution using the extended Kalman filter and a close coupling approach. The trajectories thus obtained were compared with the original reference ones in search of discrepancies. Table 9 confronts the target requirements for possible applications - mapping, precision agriculture - and the performance of the GINSEC algorithm. These results showed that the resulting navigation solution is compliant with the (hardest) application requirements the GINSEC project is targeted at.

The former steps served as a starting check point to validate the expected results using simulation techniques only. As soon as a prototype board assembling both low-cost IMUs and GNSS receiver was available, real tests took place. In this case, the prototype board was assembled together with a high quality reference platform, so data for both systems could be collected simultaneously in the same test conditions.

The reference platform consisted of an iMAR FJI IMU plus a Javad TR-G3T geodetic-grade GNSS receiver, a setup repeatedly used in other projects because of its proven quality. See Table 10 for details.

\begin{tabular}{|l|c|c|c|c|}
\hline Component & \multicolumn{2}{|c|}{ Mean } & \multicolumn{2}{c|}{ Standard deviation } \\
\hline & Achieved & Target & Achieved & Target \\
E, N (m) & 0.27 & 2.00 & 0.210 & 0.400 \\
U (m) & 0.98 & 1.00 & 0.200 & 0.400 \\
$\begin{array}{l}\text { Levelling } \\
\text { (deg) }\end{array}$ & 0.06 & 0.50 & 0.021 & 0.020 \\
$\begin{array}{l}\text { Heading } \\
\text { (deg) }\end{array}$ & 0.11 & 2.00 & 0.025 & 0.020 \\
\hline
\end{tabular}

Table 9: Achieved and target requirements for typical professional applications

\begin{tabular}{|l|l|}
\hline \multicolumn{2}{|c|}{ iMAR FJI / Javad TR-G3T precision } \\
\hline Horizontal precision & $0.02(\mathrm{~m})$ \\
Vertical precision & $0.01(\mathrm{~m})$ \\
Heading precision & $\leq 0.01(\mathrm{deg} / \mathrm{h})$ \\
Pitch/Roll precision & $\leq 0.005(\mathrm{deg} / \mathrm{h})$ \\
\hline
\end{tabular}

Table 10: Precision of the combined iMAR FJI / Javad TR-G3T reference system

A van was used to perform the dynamic (terrestrial) tests. Both the prototype board and the reference system - rigidly attached to each other to avoid undesired changes in their relative positions - were mounted on the van. Several tests were performed, collecting a number of real data sets.

These were processed with NAVEGA in the same way described above. The trajectory obtained by the high quality system was considered as the reference one, that is, the results obtained from data coming from the prototype board were to be compared with this reference trajectory.
The results obtained after processing real data were completely coherent with those computed in the simulation phase (see again Table 9) so are not reproduced here.

The most noticeable difference between simulated and real data sets is that, in the first case, very high dynamics trajectories where put to the test, thanks to the use of the X-Plane flight simulator, able to generate paths that are impossible to reproduce using a van. However, the dynamics of these simulated flights are so extreme that it is difficult to imagine a useful situation where an UAV should fly loops, barrel rolls, sideslips or spins among other high-G aerobatic manoeuvres. See again Figure 8 for an example of these simulated trajectories.

These results confirmed therefore that the expectations put on the low-cost sensors together with the use of the well known closecoupling technology were realistic and that the goal of the GINSEC projects were attainable.

\subsection{Implementation on the VRBrain. Testing}

The validation of the approach described in the previous sections opened the door to its actual implementation in the target system, the VRBrain platform.

Two challenges had to be faced; the first one, to integrate the new code implementing the handling of the new sensors (IMUs, GNSS receiver) and the way the data these provided was integrated (redundancy, close-coupling) in an already existing software system, the APM autopilot. The second challenge, and probably the worst one, was to guarantee that the new code would run fluently in the target board, the VRBrain.

The APM software is very well structured; therefore, no problems arose to identify the components to modify. The implementation task was divided into several phases:

- GNSS equations and initialization of parameters,

- INS mechanization equations,

- redundant IMUs and

- RF signal propagation and phase processing equations.

The rationale behind this plan was to implement the different software components in a cumulative way that would allow testing each step taken in a gradual manner.

The VRBrain platform proved to be powerful enough as to run the modified code as fast as to guarantee the needed position update frequency, even when GNSS data was acquired - at this point, the covariance matrix of the estimated position is computed to assess its quality, which is quite costly from the computational standpoint.

Concerning testing, a rigorous software validation and verification plan, including almost 30 different tests was devised to guarantee the correctness of the software.

These tests took into account different scenarios covering all the foreseen problems and goals - i.e., multipath problems or electromagnetic interferences. Static tests (laboratory) were addressed to provide with a way to check the correctness of the aforementioned development phases in the implementation of the full navigation algorithm. Dynamic tests (terrestrial vehicles, and especially, the UAV) would take place only once the full algorithmic implementation was finished. 
Most of these tests were addressed to check that the new navigation solution met the goals targeted at the improvement of the autopilot's behaviour, which of course, is an important requisite to accomplish. Perhaps, however, one of the most noticeable tests from the SMEs standpoint is the one devised to check how the quality of such navigation solution would affect the quality of the products derived from the payload carried by the UAV.

Showing that these products meet a certain specifications is the keystone to certify that the new system is qualified for production tasks in markets where these requisites are a must.

This test was devised as a classic photogrammetric flight, where images were to be captured using a camera as payload. The orientation (position and attitude) of these images would be computed by means of two independent techniques:

- using the classic bundle block adjustment, which does not rely at all on the navigation data captured by the UAV and,

- Computing the orientation of these images using the information included in the navigation solution.

The comparison between the results obtained by these two methods provides with an objective and quantifiable mechanism to assess the quality of the prototype.

Although not fully finished yet, the behaviour of the prototype during the different aforementioned tests showed very promising results that fit into the different set of (professional level) target applications requirements.

\section{CONCLUSIONS}

This paper presents the GINSEC project, whose target is to deliver an accurate and reliable prototype of a navigation system for the professional market based on low cost sensors (especially MEMs IMUs and GNSS receivers), rigorous sensor error modelling, data redundancy / heterogeneity, sensor geometry restrictions and a closely coupled hybridization approach.

Although the final implementation of such concept is not yet fully tested, some preliminary results are very encouraging; (at least some) low-cost MEMS sensors behave almost as well as other more expensive counterparts, the computation of the navigation solution for both simulated and real data using the specialized, well-seasoned GEMMA system produced results that show its feasibility and suitability for the stated purposes; the dual-GNSS based heading algorithm showed that acceptable results could be obtained for long baselines and the ongoing research seems to point to the same direction to those below 1 metre; finally, the actual implementation of these algorithms on the target platform, the VRBrain, has gone through a quite rigorous set of tests, that, although not yet complete, seem to confirm the goodness of the ideas and development strategy behind the GINSEC project and its objectives and that this project may lead to a step beyond the current state of the art in the targeted application domain.

\section{ACKNOWLEDGEMENTS}

The research leading to these results has received funding from the European Union's Seventh Framework Programme managed by REA - Research Executive Agency (FP7/2007_2013) under Grant Agreement N.606644. We thank the European Union's REA for their support.

\section{REFERENCES}

Colomina, I., Giménez, M., Rosales, J.J. et al., 2004. Redundant IMUs for Precise Trajectory Determination. In: Proceedings of 20th ISPRS Congress, Istanbul, Turkey, pp. 12-23.

Consoli, A., Ayadi, J., Bianchi, G. et al., 2015. A multi-antenna approach for UAV's attitude determination. In: Proceedings of the 2nd IEEE International Workshop on Metrology for Aerospace, Benevento, Italy, pp. 416-420.

El-Sheimy, N., Haiying H., Xiaoji N., 2008. Analysis and modelling of inertial sensors using Allan variance. IEEE Transactions on Instrumentation and Measurement, (57)1, pp. 140-149.

Groves, P. D., 2013. Principles of GNSS, inertial, and multisensor integrated navigation systems. Artech House Publishers, Norwood,

Mendes Cóias, J. M., 2012. Attitude Determination Using Multiple L1 GPS Receivers. Instituto Superior Técnico, Technical University of Lisbon, Lisbon,

Zhen, D., 2008. Toolbox for attitude determination with a multiple-antenna system using GPS, Center for Sensor Systems (ZESS), University of Siegen, Germany.

\section{APPENDIX}

GEMMA (Generic Extensible and Modular Multisensor navigation Analysis system) is a CTTC's in-house software development. Its main goals are the precise determination of trajectories as well as the validation of new trajectory determination algorithms.

The system consist of:

- signal (measurements) generators (IMU, GNSS, odometers and magnetometers up to now,)

- filters and analysers

- trajectory generators,

- trajectory analysers and

- a generic platform for the optimal determination of trajectories (NAVEGA), the central component of GEMMA.

The main purpose of the signal and trajectory generators and filters is to provide synthetic data to test and validate new navigation algorithms. The output of the signal generators is computed according to the models defining the behaviour of the several kinds of sensors they are able to simulate. These models characterize, for instance, its error distribution.

Signal and trajectory analysers are used to characterize the error of data sets output by these sensors

NAVEGA is a software platform for the optimal determination of trajectories or paths of stochastic dynamical systems driven by observations and their associated dynamic or static models. It is a "sensor-neutral" (generic) platform, that is, makes no assumptions about how the different sensors involved in the generation of a trajectory behave. On the contrary, such behaviour is defined by means of loadable models for each sensor intervening in the computation of the output trajectory. Moreover, it is extensible. NAVEGA may handle any new sensor just by loading its model. 
Such set of tools may be used for either production or research and development purposes. For instance, NAVEGA may be used to compute a navigation solution out of the output of real sensors. This would be a production use case.

GEMMA as a whole may also, for example, be targeted at the characterization of a new sensor; the trajectory generator would create a synthetic path that would feed a signal generator (IMU, GNSS, etc.) that would produce the expected observations that such sensor would have produce in real conditions. Then, NAVEGA should be used to compute the trajectory that such observations would have originated. The analysers, later on, would be used to study the results and help to model the sensor.

Another possible use case is the validation of new trajectory determination algorithms. A typical situation would start from actual sensor data and a reference trajectory computed by a well tested navigation algorithm. NAVEGA would take these inputs and execute the new algorithm to produce the new candidate solution, which then could be compared with the reference one using the different analysis tools available. This and the previous sensor characterization example would be clear situations where GEMMA is used in development / research use cases.

GEMMA has been successfully used for several years now in a number of research projects that required a mature, reliable trajectory determination system. 